\title{
Baloxavir Marboxil Prescription and Subsequent Medical Resource Use among School-Aged Children
}

\author{
Masato Takeuchi ${ }^{1}$ and Koji Kawakami ${ }^{2}$ \\ ${ }^{1}$ Kyoto Daigaku \\ ${ }^{2}$ Kyoto University
}

May 29, 2020

\begin{abstract}
Background: Baloxavir marboxil is a novel class of antiviral agent for influenza, introduced into clinical practice in 2018. A concern remains about the variant virus with reduced susceptibility after baloxavir exposure and its clinical consequences including healthcare seeking behavior. Methods: Using a healthcare database in Japan, we compared the medical resource use following baloxavir and neuraminidase inhibitors (NAIs) treatment among children aged 7- 15 years. Study period was from December 2018 to March 2019. The primary endpoint was the composite of hospitalization, laboratory and radiological tests, and antibiotic use over 1-9 days of antiviral treatment. As prespecified exploratory analyses, secondary outcomes were assessed and subgroup analyses comparing baloxavir with each NAI were done. Results: Data from 115867 prescriptions in 115238 children were analyzed (median age: 10 years, risk of severe influenza in $26 \%$, baloxavir accounting for $43 \%$ ). Overall, baloxavir use did not increase the subsequent medical resource utilization in the composite endpoint (adjusted odds ratio [aOR]: 1.002; 95\% confidence interval $[\mathrm{CI}]: 0.999-1.005 ; \mathrm{P}=.16$ ), as were likelihoods of other secondary outcomes. In the subgroup analysis, baloxavir use was associated with higher medical resource use than oseltamivir (aOR: 1.011; 95\% CI: 1.007-1.015; P < .001) and lower resource use than zanamivir (aOR: 0.995; 95\% CI 0.991-0.999; $\mathrm{P}=.039$ ). Conclusions: Prescribing baloxavir rather than NAIs did not increase medical resource utilization within 9 days of treatment, except in one exploratory comparison with oseltamivir. Our data were derived from single-year observation in one country, thereby requiring future researches.
\end{abstract}

\section{INTRODUCTION}

Baloxavir marboxil - an inhibitor of influenza cap-dependent endonuclease - is a new class of antiviral drug against seasonal influenza [1,2]. In 2018, baloxavir was approved in Japan and the US [3]; the drug was first approved for the indication for otherwise healthy persons with uncomplicated influenza, but the indication has now expanded to persons at high-risk of influenza complications [4]. Baloxavir was estimated to have been prescribed for more than five million persons during the 2018/2019 influenza season in Japan, representing $40 \%$ of all antivirals prescribed in the country for influenza [5].

In prelicensure phase $2 / 3$ trials, the clinical efficacy of baloxavir was superior to that of a placebo and similar to that of oseltamivir, with respect to symptom duration [6]. However, viral mutations conferring less susceptibility to baloxavir - amino acid substitutions at position 38 of polymerase acidic protein - were reported in $2.2 \%$ of 182 and $9.7 \%$ of 370 baloxavir recipients in the phase 2 and 3 trials, respectively. This group of patients experienced a longer median time to alleviation of symptoms than baloxavir recipients without shedding variant strain [7]. According to surveillance data from the 2018/2019 season in Japan, $1.7 \%(6 / 343)$ of A (H1N1) strain and 9.5\% (34/357) of A (H3N2) strain exhibited reduced susceptibility to baloxavir from specimens obtained from both treated and untreated persons [8]. This raises a concern for high proportion of variant virus and changes in the clinical course of affected cases [9]. However, the clinical consequence of variant virus is not fully understood in clinical and public health perspective, due to the sparse data of this novel drug from non-trial setting. 
In this study, we hypothesized that baloxavir recipients shedding variant virus less susceptible to this drug utilized more medical resources if their symptoms were subsequently prolonged. Using a large-scale observational data in Japan, we sought to assess the association between baloxavir use and subsequent medical resource use among children, a group in which the risk of the virus developing reduced susceptibility to baloxavir is high [10].

\section{METHODS}

\subsection{Study design and setting}

This retrospective study was conducted using data provided by JMDC Inc. (Tokyo, Japan), a commercial healthcare data vendor [11,12]. The JMDC database includes data from $>100$ society-managed health insurance plans covering employees and their dependents inclusive of children. The cumulative number of enrollees since 2005 is approximately 5 million. The study period was between December 1, 2018 to March 31, 2019. In Japan, during the 2018/2019 influenza season, the dominant circulating virus was A (H3N2) strain $(56.0 \%)$, followed by A (H1N1) strain (36.5\%) and all types of B strains (7.5\%) [13]; this represented lower influenza B activity than had been observed in the preceding seasons.

This study protocol was approved by the ethics committee at Kyoto University, including waiver of informed consent from each patient.

\subsection{Data source}

JMDC data includes patient-level demographic information, inpatient and outpatient data - such as diagnosis and procedures - and prescriptions. Claims data are collected from clinics, hospitals and pharmacies; these records are traceable even when an enrollee utilizes different healthcare providers. Prescriptions, procedures, admissions, and the beginning of medical care are indexed by date. All diagnoses are coded using International Classification of the Diseases, Tenth Revision (ICD-10) code, along with Japanese standardized diagnostic codes. The latter provide more detailed information than ICD-10 code, such as influenza virus type (eg, type A, type B or unspecified). All prescriptions refer Anatomical Therapeutic Chemical Classification System code together with brand name, and procedures are encoded using Japanese-specific procedure codes.

\section{3 Patients}

We included children aged 7-15 years who were prescribed antiviral drugs for influenza in an outpatient setting during the 2018/2019 influenza season (December 1, 2018 to March 31, 2019). During this period, baloxavir was approved for children whose body weight was [?]10kg without age restriction, and was available only as a tablet; we nonetheless did not include young children in this study, assuming that they would have had difficulty in swallowing a pill. The patients were included into the cohort by the date of prescription (the index date), and were followed 1-9 days after the index date; this window was selected as comparable to those of baloxavir clinical trials $[6,14]$. If a patient received repeated prescriptions for antivirals after this period ended, we regarded as the event as a separate infection and entered the patient into the cohort again.

Underlying comorbidities conferring a high risk of influenza complications were examined for each patient by ICD-10 code. These included asthma or chronic lung diseases, metabolic and endocrine disorders, neurological and neurodevelopmental disorders, heart diseases, and blood disorders (Supplemental Materials). These comorbidities were prespecified, harmonizing the eligibility criteria of CAPSTONE-2 trial (a phase 3 baloxavir trial conducted exclusively in patients at risk of severe influenza) and selected literature relevant to complications of influenza infection [14-16]. To correctly specify the presence of these comorbidities, patients with JMDC data history $<12$ months before the index date were excluded from the study.

\subsection{Exposure}

The primary exposure of interest was baloxavir prescription. We selected oral and inhaled neuraminidase inhibitors (NAIs) as the active comparator, namely oseltamivir, zanamivir and laninamivir. Intravenous NAI treatment was not considered as a comparator because recipient for this form might have the different 
characteristic (eg, severity) from those receiving baloxavir and other forms of NAIs. Within the same disease course, a few patients $(<0.1 \%)$ received a different type of antiviral drug or more than one type. We assigned these patients to the antiviral group according to the first prescribed class (since the second drug was unlikely to be prescribed within 48 hours of symptom onset). All prescribed drugs, including antivirals, were identified by drug code and brand name (when necessary) from the database. All anti-influenza drugs investigated are covered by insurance for the treatment of influenza, but not covered for prevention.

\subsection{Outcomes}

The primary outcome of interest was the composite of the any of the following medical resource uses during the follow-up-period: hospitalization of all-causes, laboratory/radiological services, and systemic antibiotics use. These outcome events were identified by claims records for procedures and medications. For laboratory services, any of the complete blood count, chemical panel or rapid antigen tests were assessed. Radiological examination represented plain radiography regardless of sites. As secondary outcomes, each component of the primary outcomes and return visit to medical institutions within 9 days were compared between the baloxavir and NAIs groups. These outcomes were prespecified prior to data analysis.

\subsection{Statistical analysis}

Descriptive data are reported as the median with interquartile ranges for continuous data and numbers with percentages for categorical data. To assess the association between baloxavir use and those outcomes, we used univariate and multivariable logistic regression analyses adjusting for demographic data (age and sex) and comorbid conditions (five categories).

We conducted a prespecified subgroup analysis. Baloxavir was compared with each NAI. This analysis was planned since laninamivir is thus far licensed exclusively in Japan [17]. For this analysis, only risk-adjusted odds ratio (aOR) was reported. We also planned a subgroup analysis stratified by virus type, but influenza type B infection was only infrequently found in our data set $(<1 \%)$; consequently, we did not perform this subgroup analysis.

A $P$ value $<0.05$ was considered statistically significant. $P$ value adjustment for multiple comparisons was not conducted, and thus the findings for secondary outcomes and subgroup analysis should be viewed as exploratory, hypothesis-generating testing [18]. All analyses were done with $\mathrm{R}$ statistical environment (https://cran.r-project.org/), under version 3.61.

\section{RESULTS}

\subsection{Patient and antiviral use}

A total of 115867 prescription records of baloxavir and NAIs were identified in 115238 children. Their median age was 10 years, with interquartile ranges from 8-13 years. Males accounted for $53.3 \%$ of cases (61 396 out of 115238 ). Of these children, $26 \%$ had at least one comorbidity relevant to complicated influenza infection (Table 1).

Of all prescription records $(\mathrm{n}=115867)$, baloxavir accounted for $43.2 \%$ and, as for NAIs, oseltamivir, laninamivir, and zanamivir accounted for $16.2 \%, 28.1 \%$ and $12.5 \%$, respectively. Specific virus types were recorded in $75.1 \%$ of cases (type A: $74.2 \%$ [86 023 cases] and type B: $0.83 \%$ [964 cases]). The peak of prescription was observed in January 2019, coincident with overall influenza activity during the 2018/2019 season in Japan (Figure 1) [13].

\subsection{Outcomes}

Overall, 7,945 children consumed healthcare resources afterwards (3,469 children that received baloxavir and 4,476 that received an NAI). As compared with NAI use (Table 2), baloxavir use was not associated with increased risk of more healthcare utilization in either unadjusted (odds ratio: 1.002; 95\% confidence interval [CI]: 0.999-1.005; $P=.24$ ) or risk-adjusted (aOR: $1.002 ; 95 \%$ CI: $0.999-1.005 ; P=.16$ ) analysis. All comorbidities were small but steadily related to increased risk of medical resource use following antiviral 
treatment. We found one recorded death in a child with complex medical conditions who was treated with oseltamivir.

As for six secondary outcomes, baloxavir use was again not associated with a high likelihood of more healthcare utilization (Table 2 and Supplemental Materials). In subgroup analyses, baloxavir use was associated with higher medical resource use than oseltamivir (aOR: 1.011; 95\% CI: $1.007-1.015 ; P<$. 001) and with lower medical resource use than zanamivir (aOR: $0.995 ; 95 \%$ CI $0.991-0.999 ; P=.039$ ).

\section{DISCUSSION}

During the 2018/2019 influenza season in Japan, baloxavir was prescribed for $43 \%$ of children aged 7-15 years receiving antiviral treatment for influenza. In comparison with NAIs, baloxavir prescription was not associated with an increase in resource utilization within 9 days of treatment, with the exception of the comparison with oseltamivir.

With respect to baloxavir use and clinical consequences among children, data are limited to one published trial (including its secondary analyses), a single-arm study without an active comparator drug, and small case series reported from Japan [6,14, 19-22]. From a public health standpoint, it remains unclear whether baloxavir use and virus with reduced susceptibility to baloxavir would increase healthcare resource use; this is why we designed our study to focus on healthcare utilization rather than on the relationship between variant virus and its downstream clinical outcomes in patients.

As compared with NAIs, baloxavir prescription was not overall associated with increase in resource utilization within 9 days of treatment, except in one subgroup analysis comparing with oseltamivir $(\mathrm{aOR}=1.011 ; P$ $<.001$ ). In this subgroup analysis, we also found that baloxavir reduced resource use relative to zanamivir $(\mathrm{aOR}=.995 ; P=.039)$. In these subgroup analyses, we did not perform P-value adjustment for multiple comparisons for three reasons. First, although sophisticated statistical approaches are proposed to account for multiple comparisons, it is still controversial when and how the adjustment for multiple comparisons should be done $[23,24]$. Second, statistical testing without scientifically sound hypothesis may result in a false discovery that is difficult to explain biologically [24]. Our research hypothesis de novo was, due to less-susceptible virus, baloxavir use might result in the increased healthcare use as compared with all agents of NAI class, rather than a single NAI agent. Third, our large-scale data could detect a small but statistically significant difference that lacked clinical relevance [25]. Therefore, our subgroup analyses should be viewed as exploratory ones. However, $1 \%$ of excess (or decrease) in healthcare resource use may be substantial where baloxavir was prescribed for millions of patients in one year, such as in Japan. Future researches are accordingly warranted to assess whether our subgroup findings could be explained by chance alone or not. Until then, it should be noted that the effect of baloxavir was at best equivalent to that of oseltamivir [26].

In our study, oseltamivir accounted for about $15 \%$ of antiviral prescriptions for influenza, which proportion may be lower than in other countries. There are two plausible reasons. First, Japan is the only country to date that approved laninamivir for treatment of influenza. This long-acting NAI with single-inhalation application is sometimes preferred over a 5 -days course of oseltamivir because of its convenience [27]. Second reason is the concern for potential adverse event of oseltamivir including neuropsychiatric symptoms among teenagers [28], which negative association is currently considered unlikely [17, 29]. Aside from the low frequency of oseltamivir prescription, Japan is a top consumer of antivirals for influenza, which are commonly prescribed even for children at low risk [27]. The prescription pattern should be interpreted in this Japanese-specific context, but we believe that this issue does not affect our primary analysis, that is, the association between antiviral selection (ie, baloxavir vs NAIs) and the subsequent medical resource use.

We did not integrate return visits to healthcare providers into the primary composite outcome. In Japan, asymptomatic children recovering from influenza often revisit physicians to affirm whether they can return to school. Because this type of revisit could not be differentiated in the claims record from a visit due to continuing symptoms, return visits were only analyzed as one of the secondary outcomes. In our data, revisits occurred in $47 \%$ of children, and this event was independent of initial the antiviral class prescribed. Similarly, death was not considered an outcome because influenza-related death was expected to be infrequent among 
school-aged children.

This research describes the first-season experience of baloxavir use in routine care setting involving pediatric patients at various risk levels for severe influenza, which we believe to be the strength of our study. This study also has limitations. First, our observation was based on a single-year data with limited influenza B activity. Circulating strains and the proportion of drug-resistant virus differ year by year, and the further data are needed to ascertain whether our findings are reproducible. Second, there were a number of unmeasured factors because our data source was not constructed for research purposes; these unmeasured factors included symptom duration, the interval between symptom onset and drug use, the reason for selecting the antiviral drug prescribed, vaccination status of each children (an out-of-pocket service in Japan), and the indication of further medical resource use. Our findings might therefore be subject to bias from these effect-modifiers. Third, the generalizability of our research is unclear to other age group or other counties with different healthcare system. Fourth, whether patients adhered to the prescribed treatment is unknown. Zanamivir requires a longer treatment course than baloxavir (5 days vs. a single dose); hence, it is possible that the adherence rate was lower in this group, and this difference may contribute to higher odds of medical resource use in this group than in the baloxavir group. Finally, the accuracy of the administrative claims record is uncertain or debatable [30]. However, we believe that the exposure (defined by prescription record) and outcomes (ie, resource utilization such as hospitalization, tests and antibiotics use) were both recorded with high accuracy because of the incentive for reimbursement by the healthcare providers [31]. Further, comorbidities at high risk of severe influenza were all independently associated with future resource utilization, which may indicate that the impact of disease misclassification was minimal, if it occurred at all.

In summary, as compared with NAIs, baloxavir prescription was not overall associated with increases in healthcare resource utilization within 9 days of treatment, except in one exploratory comparison with oseltamivir. Future research efforts are warranted in broader clinical contexts; these involve the following seasons, other regions outside Japan, and the populations of different age group.

\section{REFERENCES}

1. O'Hanlon R, Shaw ML. Baloxavir marboxil: the new influenza drug on the market.

Curr Opin Virol . 2019;35:14-18.

2. Mushtaq A. Baloxavir: game-changer or much ado about nothing? Lancet Respir Med. 2018;6:903-904.

3. Mullard A. FDA approves first new flu drug in 20 years. Nat Rev Drug Discov .

$2018 ; 17: 853$.

4. Roche Group. Roche announces FDA approval of Xofluza (baloxavir marboxil) for people at high risk of developing influenza-related complications. https://www.roche.com/media/releases/med-cor-201910-18.htm. Accessed May 11, 2020

5. Ministry of Health, Labour and Welfare in Japan. Monthly supply of antiviral drug against influenza (in Japanese). https://www.mhlw.go.jp/stf/seisakunitsuite/bunya/kenkou_iryou/kenkou/kekkakukansenshou01/jichitai.html. Accessed May 11, 2020

6. Hayden FG, Sugaya N, Hirotsu N, et al. Baloxavir Marboxil for

Uncomplicated Influenza in Adults and Adolescents. N Engl J Med.2018;379:913-923.

7. Uehara T, Hayden FG, Kawaguchi K, et.al.Treatment-Emergent Influenza Variant Viruses With Reduced Baloxavir Susceptibility: Impact on Clinical and Virologic Outcomes in Uncomplicated Influenza. J Infect Dis . 2020;221:346-355

8. National Institute of Infectious Disease in Japan. Detection of antiviral drug-resistant viruses in Japan during the 2018/2019 influenza season. https://www.niid.go.jp/niid/images/flu/resistance/20191128/dr1819e20191126-1.pdf. Accessed May 11, 2020 
9. Gubareva LV, Fry AM. Baloxavir and Treatment-Emergent Resistance: Public

Health Insights and Next Steps. J Infect Dis .2020;221:337-339

10. Omoto S, Speranzini V, Hashimoto T, et al. Characterization of influenza virus

variants induced by treatment with the endonuclease inhibitor baloxavir marboxil.

Sci Rep . 2018;8:9633.

11. Kasamo S, Takeuchi M, Ikuno M et al. Real-world pharmacological treatment patterns of patients with young-onset Parkinson's disease in Japan. J Neurol . 2019;266:1944-1952.

12. Suchard MA, Schuemie MJ, Krumholz HM, et al. Comprehensive comparative effectiveness and safety of first-line antihypertensive drug classes: a systematic, multinational, large-scale analysis.Lancet . 2019;394:1816-1826.

13. National Institute of Infectious Disease in Japan. Infectious Agents Surveillance Report. https://nesid4g.mhlw.go.jp/Byogentai/Pdf/data2j.pdf (in Japanese). Accessed May 11, 2020

14. ClinicalTrials.gov. Study of S-033188 (Baloxavir Marboxil) Compared With Placebo or Oseltamivir in Patients With Influenza at High Risk of Influenza Complications (CAPSTONE 2). https://clinicaltrials.gov/ct2/show/NCT02949011. Accessed May 11, 2020

15. Cromer D, van Hoek AJ, Jit M, Edmunds WJ, Fleming D, Miller E. The burden of

influenza in England by age and clinical risk group: a statistical analysis to

inform vaccine policy. J Infect. 2014;68(4):363-371.

16. Hardelid P, Verfuerden M, McMenamin J, Gilbert R. Risk factors for admission

to hospital with laboratory-confirmed influenza in young children: birth cohort

study. Eur Respir J. 2017;50. pii: 1700489.

17. Bassetti M, Castaldo N, Carnelutti A. Neuraminidase inhibitors as a strategy

for influenza treatment: pros, cons and future perspectives.

Expert Opin

Pharmacother. 2019;20:1711-1718.

18. Sun X, Ioannidis JP, Agoritsas T, Alba AC, Guyatt G. How to use a subgroup

analysis: users' guide to the medical literature. JAMA . 2014;311(4):405-411.

19. Hirotsu N, Sakaguchi H, Sato C, et al. Baloxavir marboxil in Japanese

pediatric patients with influenza: safety and clinical and virologic outcomes.

Clin Infect Dis. 2019. doi: 10.1093/cid/ciz908. [Online ahead of print]

20. Kakuya F, Haga S, Okubo H, Fujiyasu H, Kinebuchi T. Effectiveness of baloxavir

marboxil against influenza in children. Pediatr Int.2019;61(6):616-618.

21. Sato M, Takashita E, Katayose M, Nemoto K, Sakai N, Hashimoto K, et al. Clinical and Virological Efficacy of Baloxavir Marboxil in children with Influenza A. In: Options X for the Control of Influenza . Singapore: International Society for Influenza and Other Respiratory Virus Diseases; 2019, Abstract 10510

22. Saito R, Osada H, Chon I, Noshi T, Hara K, Sato I, et al. Clinical effectiveness of baloxavir marboxil compared to oseltamivir - appearance of mutated viruses at position 38 in PA protein for influenza 
A/H1N1pdm09 and A/H3N2. In: Options $X$ for the Control of Influenza. Singapore: International Society for Influenza and Other Respiratory Virus Diseases; 2019, Abstract 11026

23. Althouse AD. Adjust for Multiple Comparisons? It's Not That Simple.Ann Thorac Surg . 2016;101:16441645 .

24. Rothman KJ. Six persistent research misconceptions. J Gen Intern Med. 2014;29:1060-1064.

25. Zhu VZ, Tuggle CT, Au AF. Promise and Limitations of Big Data Research in

Plastic Surgery. Ann Plast Surg . 2016;76(4):453-458.

26. Hawkes N. Sixty seconds on . . . baloxavir. BMJ . 2018;363:k4531.

27: Zaraket H, Saito R. Japanese Surveillance Systems and Treatment for Influenza.

Curr Treat Options Infect Dis. 2016;8:311-328.

28. Urushihara H, Doi Y, Arai M, et al.. Oseltamivir prescription and regulatory actions vis-à-vis abnormal behavior risk in Japan: drug utilization study using a nationwide pharmacy database. PLoS One . 2011;6:e28483.

29. Harrington R, Adimadhyam S, Lee TA, Schumock GT, Antoon JW. The Relationship Between Oseltamivir and Suicide in Pediatric Patients. Ann Fam Med . 2018;16:145-148.

30. Cook JA, Collins GS. The rise of big clinical databases. Br J Surg . 2015;102:e93-e101.

31. Harbaugh CM, Cooper JN. Administrative databases. Semin Pediatr Surg . 2018;27:353-360.

Table 1. Patient characteristics and outcomes ${ }^{+}$

\begin{tabular}{|c|c|c|c|}
\hline Variable & All $(\mathrm{n}=115,238)$ & Baloxavir $(\mathrm{n}=49,872)$ & $\begin{array}{l}\text { Neuraminidase } \\
\text { inhibitors }(\mathrm{n}=65,524)\end{array}$ \\
\hline \multicolumn{4}{|l|}{ Demographics } \\
\hline $\begin{array}{l}\text { Age, median } \\
\text { (interquartile range), y }\end{array}$ & $10(8-13)$ & $11(9-13)$ & $10(8-12)$ \\
\hline Male sex, N (\%) & $61,396(53.3)$ & $26,724(53.6)$ & $34,763(53.1)$ \\
\hline $\begin{array}{l}\text { Asthma/chronic lung } \\
\text { disease }\end{array}$ & $24,638(21.4)$ & $10,797(21.7)$ & $13,879(21.1)$ \\
\hline $\begin{array}{l}\text { Metabolic/endocrine } \\
\text { disorder }\end{array}$ & $2,308(2.0)$ & $954(1.9)$ & $1,357(2.1)$ \\
\hline Neurological condition & $2,553(2.2)$ & $1,055(2.1)$ & $1,502(2.3)$ \\
\hline Heart disease & $1,990(1.7)$ & $816(1.6)$ & $1,178(1.8)$ \\
\hline Blood disease & $2,477(2.1)$ & $1,022(2.0)$ & $1,458(2.2)$ \\
\hline \multicolumn{2}{|l|}{ Outcome, N (\%) ${ }^{++}$} & $13,336(26.7)$ & $17,211(26.3)$ \\
\hline Composite & $7,945(6.9)$ & $3,451(6.9)$ & $4,474(6.8)$ \\
\hline Hospitalization & $17(0.01)$ & $6(0.01)$ & $11(0.02)$ \\
\hline Laboratory test & $6,671(5.8)$ & $2,876(5.8)$ & $3,810(5.8)$ \\
\hline Imaging & $1,741(1.5)$ & $787(1.4)$ & $958(1.5)$ \\
\hline Antibiotics use & $3,490(3.0)$ & $1,488(3.1)$ & $2,008(3.1)$ \\
\hline Return visit $\$$ & $54,724(47.2)$ & $23,688(47.3)$ & $31,036(47.4)$ \\
\hline
\end{tabular}

${ }^{+}$During the season, 628 children received influenza treatment more than once; baloxavir users and neuraminidase inhibitor users were not mutually exclusive. ${ }^{++}$Denominator was the total number of prescrip- 
tions (ie, 115,867, 50,047, and 65,820 for all patients, baloxavir users, and neuraminidase inhibitor users, respectively). ${ }^{\S}$ Not included in the primary composite endpoint.

Table 2: Outcomes and Comorbidities

\begin{tabular}{|c|c|c|}
\hline Analysis and variable & $\begin{array}{l}\text { Hazard ratio }(95 \% \\
\text { confidence interval) }\end{array}$ & $P$ value \\
\hline \multicolumn{3}{|l|}{ Primary analysis } \\
\hline Composite (univariate) ${ }^{++}$ & $1.002(0.999-1.005)$ & .24 \\
\hline 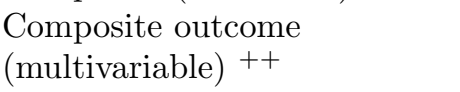 & $1.002(0.999-1.005)$ & .16 \\
\hline Age (per 5-year increase) & $0.995(0.992-0.998)$ & $<.001$ \\
\hline Sex (male) & $1.002(0.999-1.005)$ & .15 \\
\hline Asthma/chronic lung disease & $1.059(1.056-1.063)$ & $<.001$ \\
\hline Metabolic/endocrine disorder & $1.021(1.010-1.031)$ & $<.001$ \\
\hline Neurological condition & $1.025(1.015-1.035)$ & $<.001$ \\
\hline Heart disease & $1.013(1.002-1.024)$ & .020 \\
\hline Blood disease & $1.016(1.005-1.027)$ & .002 \\
\hline \multicolumn{3}{|l|}{ Secondary outcome s $^{\S}$} \\
\hline Hospitalization $^{++}$ & $1.000(1.000-1.000)$ & .77 \\
\hline Laboratory test ${ }^{++}$ & $1.000(0.998-1.003)$ & .78 \\
\hline Imaging $^{++}$ & $1.000(0.999-1.002)$ & .16 \\
\hline Antibiotics use ${ }^{++}$ & $1.000(0.998-1.002)$ & .67 \\
\hline Return visit ${ }^{++}, \mathbb{I}$ & $1.005(0.999-1.011)$ & .082 \\
\hline \multicolumn{3}{|l|}{ Subgroup analysis } \\
\hline Oseltamivir ${ }^{++}$ & $1.011(1.007-1.015)$ & $<.001$ \\
\hline Laninamivir ${ }^{++}$ & $0.999(0.996-1.003)$ & .81 \\
\hline Zanamivir $^{++}$ & $0.995(0.991-0.999)$ & .039 \\
\hline
\end{tabular}

${ }^{+}$Hazard ratio represented to the third decimal point. ${ }^{++} \mathrm{A}$ hazard ratio $>1$ indicates that baloxavir increased the odds of healthcare utilization. ${ }^{\S}$ Adjusted for demographic characteristics and comorbidities. ${ }^{\mathbb{I}}$ Not included in the primary composite endpoint.

Figure Legend

Figure 1: Trend of Antiviral Prescription and Influenza Activity in Japan

Disease activity during the 2018/2019 influenza season in Japan, reproduced from https://www.niid.go.jp/niid/en/survaillance-data-table-english.html (National Institute of Infectious Disease in Japan. IDWR Surveillance Data Table. Accessed May 11, 2020). Flu: influenza, NAI: neuraminidase inhibitors

\section{List of Supporting information}

Supplemental Table 1

Supplemental Table 2

\section{Data sharing and data accessibility}

The data that support the findings of this study are available from JMDC. Restrictions apply to the availability of these data, which were used under license for this study. 


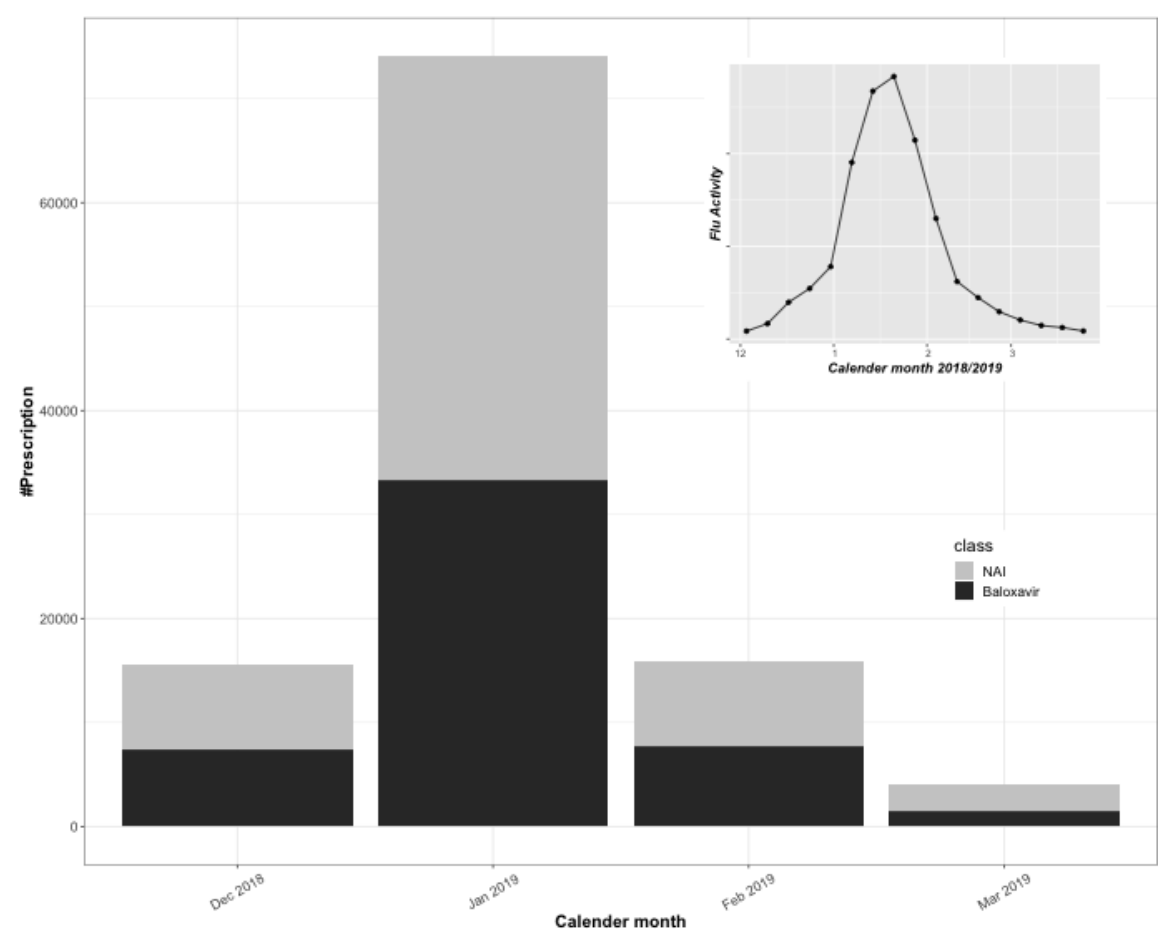

